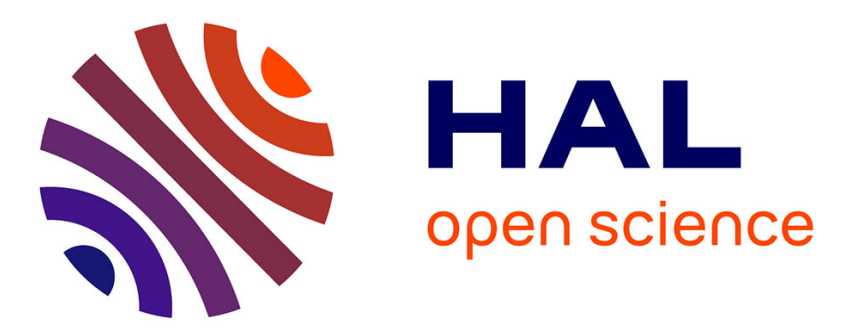

\title{
Doubaï : ville du pétrole ou projet métropolitain post-pétrolier?
}

Brigitte Dumortier, Marc Lavergne

\section{To cite this version:}

Brigitte Dumortier, Marc Lavergne. Doubaï : ville du pétrole ou projet métropolitain post-pétrolier?. Annales de géographie, 2002, $\mathrm{n}^{\circ} 623$ (623), pp.41-59. hal-00398972

\section{HAL Id: hal-00398972}

https://hal-confremo.archives-ouvertes.fr/hal-00398972

Submitted on 25 Jun 2009

HAL is a multi-disciplinary open access archive for the deposit and dissemination of scientific research documents, whether they are published or not. The documents may come from teaching and research institutions in France or abroad, or from public or private research centers.
L'archive ouverte pluridisciplinaire HAL, est destinée au dépôt et à la diffusion de documents scientifiques de niveau recherche, publiés ou non, émanant des établissements d'enseignement et de recherche français ou étrangers, des laboratoires publics ou privés. 


\title{
Doubaï : ville du pétrole ou projet métropolitain post-pétrolier ?
}

\author{
Brigitte Dumortier \\ (université de Paris IV-Sorbonne) \\ Marc Lavergne
}

(CNRS /université de Paris X-Nanterre)

\begin{abstract}
Résumé
Sur la rive arabe du golfe Persique, Doubaï est la seconde ville des Emirats Arabes Unis, après AbouDhabi, la capitale fédérale. Depuis une trentaine d'années, le devenir de Doubaï est lié à l'exploitation des hydrocarbures, directement, car l'émirat dispose des modestes revenus pétroliers de quelques gisements bientôt épuisés, et indirectement, puisqu'il profite, par le biais du budget fédéral, des immenses ressources de 1'Emirat d'Abou-Dhabi. Mais Doubaï est aussi une cité-marchande, héritière d'une longue tradition maritime fondée sur la pêche perlière et la navigation commerciale, partie prenante dans la mondialisation comme centre de transit et de redistribution vers des horizons qui dépassent désormais ses liens traditionnels avec l'Iran et le sous-continent indien.
\end{abstract}

Cet article s'efforce de montrer, sans celer les ombres au tableau, que Doubaï n'est pas une simple ville pétrolière du Golfe, Babel futuriste bâtie sur du sable, mais une métropole post-pétrolière, édifiée sur un projet économique clairvoyant qui intègre habilement le passé pré-pétrolier pour en faire un atout dans la compétition internationale

Mots-clés : Golfe persique, Emirats Arabes Unis, Doubaï, mondialisation, métropolisation, zones franches, pétrole

\begin{abstract}
On the Arab shore of the Persian Gulf, Dubai is the second most important town in the United Arab Emirates, the first one being the federal capital, Abu Dhabi. For 30 years Dubai economic development has been directly and indirectly linked to oil. But Dubai is also a merchant city with a maritime tradition founded on pearl-fishing and sea-trade. It has become a center for transit and reexportation not only to its traditionnal partners as Iran or India, but to more and more numerous, various and distant countries.

The aim of this article is to appreciate the extent to which Dubai is an oil city of the Gulf and/or an emerging post oil metropolis.

Key-words : Persian Gulf, United Arab Emirates, Dubai, globalisation ,free zones, petroleum
\end{abstract}

Doubaï, moderne Eldorado, qui dresse ses tours futuristes sur les rivages désolés du golfe Persique, est devenue en quelques décennies un kaléidoscope humain dont l'attrait, loin de se démentir, s'amplifie. Qu'est-ce qui peut faire courir ainsi le monde entier vers cette côte du 
Golfe, écrasée de chaleur moite, naguère encore confinée à l'écart de toutes les grandes voies de commerce intercontinentales?

C'est grâce, semble-t-il, à une alchimie rare, née à la fois de la capacité de forces endogènes à exploiter des virtualités et à la nécessité d'un pôle économique de ce type, au niveau régional et mondial, que le mythe est devenu réalité. Il ne s'agit donc pas d'une construction urbaine engendrée par la population locale, selon les modes courants de l'exode rural . Doubaï correspond bien plus à un projet, qui se donne à lire dans sa croissance, son architecture, son urbanisme et ses plans de développement et se met en scène à travers ses tours, ses hôtels et ses bars, ses golfs et ses plages, ses salles de congrès et ses chaînes de télévision.

Nous tenterons sinon de déchiffrer ce message, du moins d'esquisser des pistes : derrière le pétrole aujourd'hui, les perles hier, l'or encore, quel est le moteur du succès de la ville, quelle est la condition de ses habitants, autochtones et étrangers, comment s'agence cette mosaïque sociale, ethnique et culturelle?

\section{Fortune et infortunes d'une ville pétrolière}

\section{Une urbanité inachevée}

Doubaï est une ville-champignon passée de 10000 hab.en 1900 à 778000 en 1998. Sa population a plus que décuplé en quinze ans, à dater du début de l'exploitation du pétrole : en 1968 elle ne comptait encore que 59000 hab. et atteignait déjà 611000 hab. en 1993. Les chiffres suivants illustrent le rythme de la croissance urbaine : 183 000 hab. en 1975, 419000 en 1985, 674100 en 1995, selon les estimations officielles. Le cas de Doubaï n'est pas exceptionnel dans cette région du monde : toutes les bourgades du Golfe qui ont bénéficié de la manne pétrolière ont connu de pareils taux de croissance au $\mathrm{XX}^{\mathrm{e}}$ siècle. Abou Dhabi, capitale politique et principal producteur de pétrole de la Fédération des Émirats Arabes Unis, a connu une croissance de même ampleur Doubaï appartient également au paradigme des villes pétrolières du Golfe, en ce que sa croissance est due à un afflux de travailleurs immigrés. Ceux-ci, attirés par les besoins d'une économie délibérément mise en chantier par ses promoteurs, représentent $85 \%$ de la population, et sont en majorité, 
originaires d'une aire culturelle différente, le sous-continent indien. Mais, à la différence d'Abou Dhabi en particulier, Doubaï s'est transformée en une grande ville, pôle régional, voire mondial, des affaires, sans avoir bénéficié de ressources pétrolières considérables (les ressources propres en hydrocarbures seront épuisées d'ici 20102020), ni accédé au statut de capitale fédérale. D'autre part, sa croissance rapide a été parfaitement maîtrisée, à la fois dans l'urbanisme et dans le fonctionnement social, en dépit de ce déséquilibre démographique entre nationaux et étrangers.

Doubaï est une ville d'argent: la possibilité d'en gagner explique seule la concentration humaine qui s'est faite sur ce rivage inhospitalier du golfe Persique. L'appât du gain est la clé du fonctionnement d'une société très diverse et stratifiée, qui ne compose pas (pas encore ?) un ensemble cosmopolite, au sens où on l'entendait des emporium ottomans de la Méditerranée orientale, qui ont fait naître une civilisation propre, faite de mélanges. À Doubaï il s'agit plutôt d'une mosaïque de groupes humains aux fonctions parfois spécialisées dont l'articulation professionnelle est génératrice de richesses, mais dont la vie sociale commune est absente ou réduite au minimum. Un exemple de l'agrégation de ces groupes humains à la mosaïque (et non au creuset) dubaïote est fourni par l'irruption des citoyens de la CEI ${ }^{1}$. Le même rôle d'emporium est joué par Doubaï visà-vis des commerçants d'Afrique au sud du Sahara ; venus d'abord de Somalie, ils affluent désormais de pays aussi lointains que la Côte d'Ivoire ou le Congo pour faire leurs achats, en s'appuyant sur des proto-réseaux, plus ou moins élaborés, de négociants installés à Doubaï

Doubaï est encore une ville de passage, à peu près exempte de territorialisation; la question reste ouverte de savoir si la succession des individus qui y vivent, pour des durées plus ou moins longues (de quelques années à plusieurs générations) y enracine toutefois des modèles de comportements sociaux, qui permettraient d'en esquisser une étude sociologique, par delà les comportements individuels

Les nationaux et les premiers habitants de la ville ont certes un attachement plus long au lieu; pour certains, leurs parents en ont construit les premières maisons, ouvert les premières boutiques... Il faut pourtant se garder de schématiser : tous les nationaux ne sont pas des citadins de souche ; ils peuvent provenir de tribus bédouines de l'intérieur, attirées par la prospérité de la ville, tandis que les

1. Cf. Arabies, «Doubaï, l'irrésistible ascension d'un magazine en langue russe »,janvier 1998, pp.56-57 
descendants des premiers commerçants iraniens ou indiens peuvent légitimement considérer que leurs ancêtres comptent parmi les plus anciens habitants ${ }^{2}$. Quoi qu'il en soit, les nationaux constituent une catégorie à part de la population, jouissant de la rente pétrolière, au sein d'un État-providence qui pourvoit à leurs besoins essentiels. Mais qu'en est-il de leur vie quotidienne, de leurs lieux et modes de sociabilité, des valeurs qui sous-tendent leurs actes? En quoi se plient-ils à un urbanisme, à une architecture, à une société même qui leur est imposée, et dans quelle mesure sont-ils, au contraire, les maîtres de leur environnement? Sont-ils un groupe indifférencié, ou bien, comme on peut le supposer, sont-ils traversés par ces clivages lignagers et tribaux qui structurent toute société arabe? En particulier, y a-t-il parmi eux des groupes ou des individus ayant le monopole de la prise de décision, tandis que d'autres seraient cantonnés dans un rôle de consommateurs silencieux?

Quant aux immigrés, leur palette est encore plus variée : diversité des origines et des cultures, certes ; mais aussi des conditions socioéconomiques, des statuts juridiques, de la compétence à s'exprimer, c'est-à-dire au total diversité des degrés et des modes d'appartenance à la ville et d'appropriation de la ville, selon le mode de logement (du baraquement en tôle non climatisé à la villa, en passant par l'appartement ou la chambre d'hôtel) et aussi selon la situation de famille.

Pour donner une idée forcément schématisée de ce kaléidoscope humain qu'est la société de Doubaï, il est tentant d'associer chaque communauté à un domaine d'activité. On dirait alors que les magasins sont souvent tenus par des Indo-Pakistanais, l'administration par des Arabes (Égyptiens, Soudanais, Palestino-Jordaniens principalement), que les affaires sont détenues par de grandes familles d'origine indienne ou persane, que l'armée est baloutche avec un encadrement jordanien ou britannique, tandis que les grandes entreprises occidentales ou extrême-orientales, représentées par des expatriés, emploient des cadres libanais ou indiens, et des ouvriers coréens, thaïlandais ou philippins.

La réalité est infiniment plus complexe et mouvante que ces généralisations souvent reprises, mais non étayées en l'absence de sources statistiques accessibles et fiables: la quantification des mouvements migratoires est un sujet politiquement sensible, sans

2. Au début du XXe siècle, J.G. Lorimer (Gazetteer of the Persian Gulf and Central Arabia, vol.2, Superintendent Government Office Printing, Calcutta, 1915) recense à Doubaï 200 maisons de Baloutches, 250 de Persans, 67 individus originaires des Indes, et 23 Khojas ; leurs descendants ont la nationalité émirienne. 
parler, en dépit d'un législation très stricte sur les visas et les permis de travail, d'une immigration clandestine par définition difficile à cerner et de la pratique courante des séjours prolongés au-delà de leur durée légale. Si l'on se risque à élaborer une typologie croisant nationalité et activité, le simple vécu quotidien montre à quel point chaque entreprise, chaque service public est à la fois le lieu de rencontre et le champ clos des rivalités entre les différentes nationalités qui s'y côtoient.

Aborder la question en esquissant des monographies par nationalités ne fait que renforcer cette complexité. La communauté indienne, dont la supériorité numérique perdure, présente une stratification sociale marquée, des grandes familles marchandes ayant pignon sur rue au petit tailleur du souk rivé à sa machine à coudre, des cadres intermédiaires du secteur bancaire aux travailleurs nonqualifiés de passage, sous contrat collectif. Du fait de l'existence de filières migratoires et de fortes disparités internes en Inde, travailler par communauté s'avère plus pertinent. Il est patent que les emplois occupés par les Kéralites, appréciés dans les services domestiques n'ont rien à voir avec ceux des Sikhs, prisés pour les travaux de force.

Les travailleurs du sous-continent indien situés en bas de l'échelle sociale n'ont aucun droit, ils ne sont guère considérés que comme une force de travail, souvent surexploitée. La pire des situations est celle des employées domestiques sri-lankaises, dont le statut, comme partout ailleurs au Moyen-Orient arabe où elles sont présentes, s'apparente à l'esclavage dans certaines familles. Ces habitants ont pourtant une existence sociale : ils occupent l'espace domestique ou les chantiers, et parfois l'espace public (sorties dominicales à la messe pour les employés philippins ou originaires du Sud de l'Inde, sorties au cinéma, pour rêver devant les productions des studios de Madras ou de Bombay, sorties au marché...).

Il serait donc très réducteur de ne voir en Doubaï qu'un lieu désincarné, point de rencontre de l'argent, de la main-d'œuvre et de l'énergie bon marché. Si effectivement, ces trois éléments sont les clés du succès de la ville, ne doit-on pas rechercher également si un certain esprit $d u$ lieu ,différenciant Doubaï de ses homologues du Golfe, n'y aurait pas sa part? Doubaï apparaît comme une ville de juxtapositions et de mélanges, de lieux et d'activités affichés comme «traditionnels» ou comme "modernes », dont on peut se demander s'ils s'opposent ou se complètent. Quel lien ou quelle volonté peut bien assurer leur coexistence?

\section{Un espace urbain en chantier}


Doubaï est née de part et d'autre d'un méandre à l'embouchure du $k h o r^{3}$ de Doubaï ( se reporter à la carte 1 pour le repérage des quartiers). Deux villages s'individualisent ainsi dès l'origine : Doubaï proprement dit au sud et Deira au nord. Deux ponts, Al Garoud et Al Maktoum, complétés par un tunnel sous l'embouchure, permettent aux véhicules de passer d'une rive à l'autre de la Crique, animée par le va-et-vient incessant des abras, embarcations qui assurent le transbordement des piétons pour quelques fils (un dirham, soit 100 fils, vaut moins de deux francs).

Par la Crique, la ville a accès aux eaux du Golfe, tout en étant abritée. Le site est d'occupation ancienne, et même antique ${ }^{4}$, mais il ne faudrait pas pour autant attribuer à l'occupation actuelle les vertus héritées sans solution de continuité de cette antiquité. En réalité, le site n'a été occupé définitivement qu'au XIXe siècle, par des Bédouins originaires des oasis du Liwa, qui cherchaient un complément saisonnier de ressources halieutiques et pratiquaient aussi la pêche perlière.

Après la signature des Accords de la Trêve avec les chefs tribaux qui s'adonnaient à la piraterie, l'installation d'un Résident britannique dépendant de Bombay permit, paradoxalement, la floraison d'une intense contrebande d'or en direction de l'empire des Indes ${ }^{5}$. Cette histoire de pirates, de perles et d'or exhale encore des parfums d'aventure coloniale aux confins d'un Orient mystérieux.

\section{Le noyau ancien, cour d'une citadinité ouverte sur l'espace régional}

Aujourd'hui, cette époque est encore incarnée par les vieux souks de Doubaï et de Deira.(photo 1). Mais loin d'être muséifiés, malgré la rénovation dont ils ont fait l'objet, ces marchés resserrés dans quelques allées piétonnes, ombragées de claires-voies en bois, restent d'intenses lieux de vie et d'activité marchande. Ils présentent une forte spécialisation (souk des épices, souk de l'or, souk des tissus) que l'on retrouve dans certaines rues (magasins de matériel électronique de la rue Al-Fahidi) ou certains centres commerciaux (tapis de la Deira Tower). L'activité trépidante qui s'y déploie, l'afflux de toutes

3. Mot arabe désignant généralement un cours d'eau temporaire en milieu désertique ou sahélien. Ici, étroit goulet marin pénétrant de quelques kilomètres dans l'intérieur des terres, traduit en français par "crique ".

4. D. Potts, The Arabian Gulf in Antiquity, Clarendon Press, Oxford,1990, 2 vol.

5. Si la pêche perlière a été ruinée par l'apparition des perles de culture japonaise vers 1930 le commerce de l'or a prospéré : Doubaï a récemment dépassé Singapour comme premier centre mondial du commerce de redistribution de l'or. Voir "Merchants of Gold ", The Middle East (TME), septembre 1998, p. 50 
les catégories de publics qui s'y pressent le soir venu, signalent le rôle à la fois économique et social de ces espaces.

Les souks et leurs abords sont le siège d'une micro-économie de petits commerces et d'échoppes de produits de consommation courante, importés pour l'essentiel, mais aussi fabriqués sur place, dans des ateliers disséminés dans la ville. Cette circulation marchande n'est pas une survivance folklorique submergée par la grande distribution omniprésente : elle offre des produits répondant à une demande locale spécifique, non standardisée, à bon marché, et s'adresse donc à la clientèle des quartiers populaires. Mais on n'est pas là dans le monde de l'informel ou du marginal ; au contraire, ce commerce de détail est souvent relié aux entreprises d'import-export et donc aux réseaux qui émanent de ou aboutissent à Taïwan, Séoul, Bombay ou Karachi. Le célèbre souk de l'or, à Deira, relève de la même culture avec ses trois cents échoppes serrées les unes contre les autres, rutilantes de bracelets et de colliers étalés à profusion .

Chargés d'humanité et d'urbanité, les souks sont aussi les lieux d'une sociabilité publique qui manque dramatiquement dans la plupart des villes de croissance récente dans la péninsule. Ici, les rapports marchands sont la base de rapports humains qui renvoient aux citadinités des métropoles de l'Inde, des cités caravanières du haut Pakistan, ou des ports du Levant. Les venelles du souk sont les rares endroits dévolus aux piétons, qui y redécouvrent les plaisirs de la promenade, de la flânerie, du lèche vitrine, des jus de fruits fraîchement pressés. Lieux de la conversation aussi, entre hommes originaires du même pays, émigrés, on dirait plutôt exilés, qui viennent téléphoner à leur famille ou changer de l'argent dans les officines privées où l'on peut s'asseoir et échanger des nouvelles en attendant son tour. Même les espaces privés comme les magasins sont des lieux de détente, où l'on peut laisser tomber le masque laborieux de la semaine.

Les berges où accostent les boutres sont avec les souks un lieu emblématique de la tradition marchande de la ville (photo 2). Les boutres, aujourd'hui encore, traversent le détroit d'Ormuz pour ravitailler soixante millions d'Iraniens sevrés d'importations pour cause d'embargo et de crise économique. Leurs flancs de bois qui cachent de puissants moteurs, sont chargés à dos d'hommes de toutes sortes de marchandises (pneus et pièces détachées, réfrigérateurs, chaînes hi-fi, téléviseurs, etc.), qui reflètent assez le caractère moderne de cette économie aux allures dépassées. 
Souks ou quais, les espaces déambulatoires du centre-ville s'ouvrent aussi sur des places, où d'autres lieux s'offrent : la mosquée, bien sûr, lieu de la convivialité et du ressourcement ; dans un autre registre, le cinéma où se recrée, comme à la mosquée, la ségrégation sexuelle. À la différence de l'Arabie saoudite, Doubaï offre à ses travailleurs immigrés originaires du sous-continent indien des cinémas urbains, à l'architecture surannée, dont les affiches offrent au regard avide des travailleurs célibataires de plantureuses actrices et leurs partenaires gominés. Le cinéma est un lieu d'attraction, même hors des heures d'ouverture; on y vient rêver devant les programmes, on s'y retrouve avant l'heure pour bavarder...

Le noyau ancien est de plus en plus «recyclé" en «centre touristique ". La carte de la ville , éditée par la Municipalité6, indique une forte concentration de "sites touristiques". Promus à ce rôle, on relève à Doubaï, le quartier de Bastakiya, le fort Al-Fahidi, le quartier du souk et, enfin, près de l'embouchure, le Palais de Cheikh Saïd alMaktoum; à Deira, sont ainsi identifiés la place des Beni Yas, confédération tribale d'Abou Dhabi d'où est originaire la dynastie des Al-Maktoum, le quartier des souks et l'école de la Ahmadiya, célèbre confrérie religieuse musulmane. La restauration du fort et du palais ainsi que leur transformation en musées de même que la réhabilitation de Bastakiyya témoignent d'une préoccupation patrimoniale nouvelle, dont la finalité semble autant identitaire que touristique.

\section{La croissance accélérée d'une ville polymorphe}

Autour de ce noyau et sur sa démolition partielle s'est développée la ville moderne, de la fin du XIXe siècle aux années 1970: cette croissance des quartiers d'habitation composés de villas ou de petits immeubles a d'abord pris une forme concentrique, à partir des deux demi-cercles initiaux de Doubaï et Deira ; en même temps, les centres d'activités se sont étendus le long du littoral et de la Crique, ainsi qu'en arrière, jusqu'aux cimetières et aux terrains vagues qui marquent la limite de cette extension urbaine.

Les grands hôtels sont les indices les plus marquants du développement des affaires à la fin des années soixante. Ils firent office de fronts d'urbanisation et restent des repères dans le paysage. Ils constituent des pôles majeurs de la vie sociale: repas d'affaires, mariages, rendez-vous personnels ou professionnels...Vecteurs d'acculturation au-delà d'une décoration à laquelle l'usage

6. Dubai tourist map, Dubai Municipality, millenium edition, 2000 
systématique des mosaïques, des tapis et des fontaines donne un cachet oriental, ils ont grandement contribué à l'importation de nouvelles habitudes alimentaires et de nouveaux modes de socialisation. À Deira, les enseignes les plus prestigieuses s'alignent sur la corniche de la Crique, tandis qu' en retrait se dispersent de petits hôtels. Le quartier des affaires et de l'administration s'étire le long de la Crique et dote Deira d'une skyline (photo 3) qui en signale l'appartenance à l'archipel de la finance mondiale. En amont du pont Maktoum, le bord de la Crique a été aménagé en un vaste parterre de verdure : le célèbre Dubaï Creek Golf Club and Yacht Club, dont les toits du Club House figurant des voiles de boutre élancées vers l'azur sont devenues l'un des emblèmes de la ville. Le golf a pour vis-à-vis sur l'autre rive le vaste parc du khor ; plus loin encore vers l'intérieur, s'élèvent épars des établissements hospitaliers, religieux ou sportifs dont les dénominations témoignent de la coexistence communautaire qui prévaut à Doubaï.

Le tournant des années 1980 a vu Doubaï préparer l'après-pétrole en se dotant d'une économie diversifiée. Cela se traduit par une expansion dévoreuse d'espace, le long d'axes stratégiques . Au nord, le littoral a été aménagé pour recevoir les souks de produits périssables : après le traditionnel marché au poisson, a été construit le souk des fruits et légumes en gros de Hamriya. Le quartier de Hamriya, dont la surface équivaut à peu près à celle de Deira, est un quartier d'habitat modeste, collectif, composé de grands immeubles alignés le long de rues rectilignes . Au-delà de la voie express qui relie Deira et Doubaï par le pont Al Maktoum et rejoint ensuite l'autoroute d'Abou Dhabi, se sont développées, sur le vaste espace désertique disponible, toutes sortes d'activités: l'aéroport international est entouré de zones industrielles et de quartiers populaires; les extensions encore en chantier ou juste tracées sur le sable, sont visibles jusque loin dans le désert..

Doubaï proprement dite a grandi de manière très différente, puisque après une zone encore en attente de lotissement, les nouveaux quartiers s'étirent entre le littoral et l'autoroute d'Abou Dhabi : Houdheiba, Mankhoul, Satwa, Al-Bada'a, puis Joumeira et AlWoussoul, Safa, Oum Suqeim et Al-Qoz. Au-delà de l'autoroute, Za'abeel fait figure de front d'urbanisation dans le désert. Cet ensemble urbain très lâche est structuré en lotissements abritant des milieux sociaux très disparates et compartimentés ; les quartiers les plus prisés sont la ligne côtière, où ont été édifiés des marinas, des wharfs, des clubs nautiques comme le Dubaï Marine, des hôtels ou des 
complexes récréatifs et hôteliers sur la plage ${ }^{7}$ C'est aussi là que la famille régnante a construit ses somptueuses résidences. Ces quartiers chics de Joumeira, Safa et Oum Suqeïm sont encore en chantier, et le tissu urbain est d'autant plus lâche que l'on s'éloigne vers le Sud. La zone industrialo-portuaire de Jebel Ali se rattache progressivement à l'agglomération de Doubaï par le développement d'infrastructures d'accueil le long du rivage où des hôtels, agrémentés d'une marina et d'un golf, servent de halte aux hommes d'affaires. Enfin, en lisière des nouveaux périmètres d'urbanisation, des fermes maraîchères se sont créées.

L'extension urbaine vers l'est reste beaucoup plus lâche et vouée pour l'essentiel aux établissements sportifs et de loisirs : hippodrome et camélodrome, terrains de golf, station d'observation de la vie amphibie et des oiseaux aquatiques au fond du khor où une vaste zone fait office de parc naturel...

\section{Une ville sans contours façonnée par l'automobile}

La ville n'a pas de limites précises, à cause du rythme effréné et des modalités de sa croissance: les constructions s'étendent sans contrainte dans le désert et l'automobile y abolit les distances. L'omniprésence de l'automobile explique le rôle structurant des autoroutes intra-urbaines et l'extension spatiale de la ville. Cette toute-puissance de la voiture, calquée sur le modèle américain, est le résultat de plusieurs facteurs convergents :

- l'enrichissement soudain de la population indigène ou immigrée, qui se traduit par un engouement pour la voiture, symbole de statut social ;

- le coût relativement bas de voitures non frappées de taxes et alimentées par un carburant extrait et produit sur place, également détaxé ;

- l'extrême chaleur ou la touffeur humide du climat, qui rend impossible la marche à pied au soleil, et impose la climatisation, dans une économie de type moderne, qui se déroule surtout dans des locaux de bureaux ou de magasins ;

- un fonds culturel qui impose la ségrégation sexuelle, la vie sociale des femmes restant en grande partie confirmée dans l'enceinte domestique et familiale. Celles-ci ont le droit de conduire, à la

7. Le Borj al Arab en forme de voile de $321 \mathrm{~m}$ de haut, exceptionnellement crédité de 7 étoiles avec ses 202 suites de grand luxe, achevé en 1998 sur une île artificielle, et son voisin de 600 chambres, le Jumeirah Beach Hotel en forme de vague de $100 \mathrm{~m}$ de haut, achevé en 1997, donnent un air obsolète à des installations qui ont à peine plus de vingt ans et ont déjà été remaniées et rénovées, comme le Chicago Beach. 
différence des femmes saoudiennes. La voiture permet aux femmes des milieux sociaux favorisés ou de classes moyennes de sortir tout en évitant de se trouver directement en contact avec le public.

L'emprise du bâti atteint aujourd'hui $25 \mathrm{~km}$ le long du trait de côte et jusqu'à $12 \mathrm{~km}$ vers l'intérieur. Au nord, au-delà de Hamriya, la césure désertique avec l'émirat voisin de Sharjah s'est estompée. Avec la ville d'Ajman, devenue un faubourg de Sharjah, l'ensemble, en voie de coalescence, tend à former une conurbation, que l'on serait tenté de qualifier de transfrontalière, où chaque ville garde sa personnalité, ses activités et, bien sûr, son autonomie politique et économique au sein des Emirats Arabes Unis, sans qu'aucune entité administrative du type "Communauté urbaine" ne soit chargée de gérer de façon concertée la croissance de ces trois capitales d'émirats limitrophes et fédérés.

Le sentiment d'un étalement sans contrainte tient aussi à des raisons administratives: la Municipalité de Doubaï a, en effet, compétence sur l'ensemble de l'Émirat et non pas sur la seule ville de Doubaï. Tout comme le toponyme Doubaï désigne à la fois l'émirat, la ville de Doubaï dans son ensemble et la rive gauche de la ville par opposition à Deira, la notion de limite de la Municipalité n'existe pas et celle-ci apparaît comme un organisme chargé de l'aménagement et de la gestion urbaine, et non pas doté de compétences autonomes par rapport à celles de la famille régnante ou du pouvoir traditionnel : un pouvoir exercé sur les hommes et non pas sur le sol8.

\section{Stratégies d'une ville post-pétrolière}

\section{Des activités mises en synergie}

Doubaï doit être envisagée, non seulement sous l'angle morphologique, mais aussi comme un organisme doté de fonctions et d'une image en grande partie indépendantes de son inscription spatiale.

L'économie urbaine repose sur trois types d'équipements :

- les pivots de l'activité tertiaire supérieure , constituant un CBD, tels le World Trade Center, la Chambre de Commerce, les tours abritant les sièges sociaux de grandes entreprises industrielles ou

8. Cf J. Walker : "Practical Problems of Boundary Delimitation in Arabia : the Case of the United Arab Emirates ", in : R.Schofield, Territorial Foundation of the Gulf States, London, UCL Press, 1996, p. 109 à 116 ; G. Joffé : " Concepts of Sovereignity in the Gulf Region, ibid.,p. 78 à 93. Voir aussi B. Dumortier, «Litiges frontaliers et revendications territoriales dans la Péninsule Arabique », in : Frontières ambivalentes, Lille, Cahiers du LGH , 1992 
financières, les hôtels internationaux, les bureaux de l'administration des affaires ;

- les zones industrielles et les grandes infrastructures de communication et de transport. comme l'aéroport international, les ports Rachid et de Jebel Ali, les autoroutes intraurbaines et interurbaines ;

- les centres touristiques et de loisirs ${ }^{9}$, c'est-à-dire les souks, centres commerciaux, grands hôtels, plages, clubs sportifs et de détente, champs de courses, parcs et jardins et golfs.

L'une des grandes forces de l'économie de Doubaï est l'étroite articulation entre ces différentes activités : les équipements de loisir servent aussi à attirer les hommes d'affaires, l'approvisionnement des centres commerciaux et des souks emplit les cargos qui font escale dans les ports, les touristes aident à rentabiliser les liaisons aériennes nécessaires aux entreprises et aux commerçants... La séparation classique entre branches d'activités n'est plus de mise dans cette économie intégrée, laissant voir de façon limpide les effets de synergie souvent masqués dans des économies plus anciennes ou plus sophistiquées: ainsi les terrains de golf ne sont pas destinés au premier chef aux résidents ou aux touristes. Certes, ils sont un élément d'attraction complémentaire pour une certaine clientèle de touristes aisés, mais ils sont surtout destinés à appâter les entreprises en quête d'un bureau régional, dont les cadres dirigeants seront sensibles à la présence de terrains de golf de qualité internationale. Pour parachever la rentabilisation de cet atout, Doubaï organise chaque année, en janvier-février, la Dubaï Desert Classic, l'une des compétitions les plus cotées du monde. Affluence garantie de sportifs et de médias, qui emplissent les hôtels, mais surtout promeuvent l'image de Doubaï dans le monde entier. On peut de la même façon évoquer la Dubaï World Cup, la course hippique la mieux dotée du monde, ou le Shopping Festival, tout à la fois opération commerciale, produit touristique, manifestation culturelle et événement médiatisé.

\section{Une polycentralité fonctionnelle}

Tandis que le noyau ancien constitue toujours un centre attractif, pour la vie sociale autant qu'économique, avec ses souks, ses mosquées, ses cinémas, son port des boutres, la ville moderne est

9. Sur le développement touristique voir TME : "Survey on Tourism : the new Explorers ", mai 1998, p. 35 à 40 et «Gulf Tourism ", mai 1997, p. 18 à 21. 
ponctuée de pôles d'activités, qui créent ou entérinent les nouvelles centralités urbaines :

- le Dubaï World Trade Center avec 39 étages, est l'un des plus hauts immeubles d'affaires du Moyen-Orient. Repère dans le paysage, à la croisée de voies autoroutières $\mathrm{N}-\mathrm{S}$ et $\mathrm{E}-\mathrm{W}$, à la frontière du Doubaï des années 1970 et des nouvelles extensions vers le sud (photo 4), il sert de blason à la réussite économique de l'émirat ;

- les centres commerciaux, belles galeries marchandes exposant tous les produits de la planète à des prix très compétitifs, ont fait la réputation de Doubaï, moderne «caverne d'Ali Baba». On peut citer à Deira l'Al-Mulla Plaza, l'Al-Ghurair Centre, le Hamarain Center, du côté de la Crique le Wafi Mall ou le Bur Juman Centre, à Joumeira le Markaz al-Joumeira, le Beach Center...

- les hôtels de luxe, implantés de préférence sur la rive nord de la Crique, ou le long du littoral vers le sud, constituent, on l'a vu, un des points forts de la vie sociale, exerçant donc une puissante attraction sur les couches aisées de la population autochtone et expatriée, avec leurs restaurants, leurs piscines, leurs bars et leurs boîtes de nuit ;

- les mosquées, dans une ville dont plus de la moitié de la population (seulement, pourrait-on dire!) est de confession musulmane, sont également des lieux de rassemblement et deviennent, lors de la grande prière du vendredi, des lieux privilégiés de sociabilité masculine.

Ces exemples de centralités exercées par des édifices ou des groupes d'édifices doivent être appréhendés en tenant compte de l'usage généralisé de la voiture, dans les couches moyennes et supérieures de la population. Pour celles-ci, la centralité est par nature multiple. Le déroulement de leurs activités au cours de la journée les amène à parcourir de grandes distances: du domicile dans une villa de Jumeira au travail dans un bureau du CBD au déjeuner dans un grand hôtel, à l'après-midi dans un club du bord de mer, aux courses du soir dans un centre commercial, chaque lieu est un centre temporaire et partiel de l'activité de certaines catégories de la population. Pour qu'un site exerce une fonction de centre à un moment donné, point n'est besoin de quartiers regroupant côte à côte de multiples fonctions complémentaires, comme dans les villes conçues avant l'ère de l'automobile. Autre conséquence, la voiture induisant un style de vie, la frontière entre la ville proprement dite et ses banlieues s'estompe, les activités pouvant se développer indépendamment de la référence à un centre unique : des hôtels de luxe, des entreprises, des centres commerciaux s'installent délibérément en périphérie, y faisant naître une animation qui s'étoffe 
progressivement et couvre la totalité du cadran horaire, à la différence de la suburbanisation américaine, ou des centres commerciaux de périphérie en Europe, animés le jour et désertés la nuit.

\section{Des infrastructures exceptionnelles}

La ville subit l'attraction, centrifuge spatialement, mais économiquement intégratrice, de plusieurs infrastructures stratégiques pour son développement. Centrales électriques et stations de désalinisation de l'eau de mer jouent un rôle clé. L'augmentation ininterrompue de la consommation implique un programme continu d'extensions. La production d'électricité et d'eau douce est concentrée de plus en plus sur le site de Jebel Ali, bien qu' en 1999 une centrale électrique ait été mise en service à Awir, à $40 \mathrm{~km}$ à l'est de la ville .

\section{Un aéroport international performant}

L'aéroport international, devenu en quelques années une plaque tournante des transports aériens régionaux et transcontinentaux, réguliers et charters, est le deuxième aéroport du Moyen-Orient : 130 destinations desservies et 9,1 millions de passagers (au départ, à l'arrivée et en transit) en 1997. C'est le deuxième du monde pour le nombre de passagers en transit (1,1 million), après celui de Narita à Tokyo.

L'aéroport symbolise également la stratégie et les conceptions de Doubaï, en ce que son activité passagers est intégrée avec un important complexe commercial hors-taxes, chargé d'attirer les voyageurs par les prix les plus alléchants et l'offre de produits la plus variée de la planète, mais aussi par des promotions et des tombolas. Cette zone franche est considérée comme la meilleure du monde, avec des ventes de 190 millions $\$$ en 199710

Quant à l'activité fret, elle est concentrée dans un "Village Cargo ", capable de traiter 250000 t/an. Créé en 1991, sur une aire de $43000 \mathrm{~m}^{2}$ dans l'enceinte de l'aéroport, il assure le transfert des marchandises importées par voie maritime d'Extrême-Orient et expédiées de là par voie aérienne vers l'Europe et les États-Unis. En 1995, environ vingt-trois compagnies aériennes utilisaient cette infrastructure, directement connectée avec les zones industrielles avoisinantes, les autoroutes qui relient Doubaï aux villes et aux États

10.TME : «Duty Free, a Shopping Revolution », mai 1998, p. 38-39. 
voisins, et les ports Rachid et Jebel Ali, mais aussi Khor Fakkan, sur le golfe d'Oman. Sept quais de fret combiné air-mer, "en font l'une des zones les plus performantes du monde pour ce type de transbordement 11 ». Ainsi, Doubaï s'est fait une spécialité des activités de réexportation, grâce à ses connections simples et rapides entre les transports aériens et maritimes. Dans ce domaine, l'activité des boutres, qui relient Doubaï à la côte iranienne, jouent un rôle prépondérant ; mais le trafic aérien intense qui relie Doubaï aux villes les plus reculées d'Asie centrale ou de l'immensité russe appartient à la même catégorie d'activités.

\section{Des ports complémentaires}

Doubaï compte quatre ports. Les anciens ports de la Crique et de Hamriya, approfondis et modernisés, fonctionnent à pleine capacité. Le port de la Crique joue un rôle important pour la redistribution vers l'Iran, l'Inde et l'Afrique orientale des cargaisons importées. En outre, sous l'impulsion décisive de Cheikh Rachid al-Maktoum, Doubaï s'est équipé avant tous ses rivaux de la région de deux ports commerciaux modernes.

Port Rachid est entré en activité en 1972 et sa capacité a été doublée à la suite du boom pétrolier de 1973. À proximité immédiate de la ville et de l'aéroport, ce port joue un rôle majeur dans l'approvisionnement de la ville, par l'importation et la réexportation de biens de consommation et de biens d'équipement. Il se prolonge au Sud par les bassins de radoub et la cale sèche pour supertankers, qui fait de Doubaï une rivale de Bahreïn pour l'entretien des pétroliers qui fréquentent le Golfe. Sa mise en service date de 1979, mais les travaux ont été achevés en 1982.

Le port de Jebel Ali a été conçu dès l'origine comme un pôle d'activité autonome, censé assurer l'avenir de l'émirat après le déclin prévu des ressources pétrolières. C'est désormais le grand port de transbordement de la péninsule arabique et des pays riverains du Golfe ; mais son activité dessert maintenant la mer Rouge, les rives de l'océan Indien et, au-delà, l'ensemble du Moyen-Orient et de l'Asie centrale. Le port a pris tout son sens lors de la création de la zone franche qui lui est rattachée : celle-ci jouit d'un statut extraterritorial qui exempte les investisseurs de toutes les charges et réglementations qui pèsent, même à Doubaï, sur l'activité économique. Fondée en

11.Roger Melki : « Doubaï l'incontournable », Arabies, 1993, p. 34 à 42. 
1983, elle permet d'échapper à l'obligation pour tout investisseur à Doubaï de réserver au moins $51 \%$ des parts à un partenaire local.

Abritant, en 2000, 1650 entreprises originaires de 85 pays, elle a permis au port de Jebel Ali de devenir le plus florissant $h u b$ régional. Ses activités de stockage, de transit et de réexportation l'emportent encore sur la production industrielle ( $35 \%$ des entreprises), qui recouvre d'ailleurs plutôt des activités de conditionnement et d'emballage qu'une véritable transformation de matières premières en produits finis. L'aire de Jebel Ali est dotée, en dehors de la zone franche, d'industries lourdes dans lesquelles l'émirat a investi des sommes considérables : usine de dessalement d'eau de mer, centrale thermique, usine d'embouteillage de gaz naturel liquéfié, usine Dubal qui produit, à partir d'alumine importée et de l'énergie à bon marché fournie par le gaz naturel, $240000 \mathrm{t}$ d'aluminium, faisant de Doubaï le premier producteur mondial d'alliages à base d'aluminium pour pièces automobiles. En 1999 a été inaugurée une usine d'engrais produisant $680 \mathrm{t} / \mathrm{j}$ d'ammoniac et $1200 \mathrm{t} / \mathrm{j}$ d'urée ${ }^{12}$.

Novateur, le port de Jebel Ali n'est cependant pas encore parvenu à maturité : l'activité industrielle sur place n'est pas encore solidement implantée et la zone franche ne tourne pas encore à pleine capacité. Les autorités cherchent désormais à attirer des industries à fort contenu capitalistique et surtout technologique, au lieu des industries simples qui y viennent prioritairement. Mais le site n'est pas encore perçu comme un lieu d'implantation possible pour les industries de l'informatique, de la télématique ou de l'électronique grand public qui ont fait la fortune des «nouveaux dragons » du sud-est asiatique. Tant que ce résultat n'aura pas été atteint, la réussite de l'entreprise demeurera fragile et il restera des sceptiques pour penser que le boom de Doubaï a été bâti sur du sable13 ( les doutes qui ont accueilli les premières affirmations de l'ambition de Doubaï14 n'ont-ils pas été, en leur temps, démentis par les faits et les chiffres? ). D'autre part, l'exemple de Jebel Ali est désormais suivi par d'autres pays : le $h u b$ de Mina Raysout à Salalah a été inauguré avec le même concept d'un port d'éclatement, qui offre l'avantage de se trouver directement en eaux libres, sur les rives de l'océan Indien. Même si la concurrence

12.Joint venture associant des capitaux privés indiens et américains avec la Emirates Trading Co (MEED 5 décembre 1997).

13. Voir des exemples de ces réserves dans MEED : "Confident that the Growth can Continue ", 5 décembre 1997, p. 34 ; Julian Taylor: "Can Dubaï survive the Global Challenge ? ", TME novembre 1998, p. 21-22.

14.Voir J.J. Tur: Les Emirats du Golfe Arabe, Que sais-je?, PUF, 1976 , "Doubaï, le superflu, mais pas l'essentiel", p.72, par exemple. Songer aussi au titre du livre de S. et J. Lacouture, Les Emirats Mirages, éditions du Seuil, 1975 
entre les deux ports est récusée et si des complémentarités sont envisagées, les entreprises actionnaires de Salalah semblent avoir opéré un choix stratégique significatif en investissant dans le nouveau port 15

La réalisation, sur le modèle du Parc Scientifique de Singapour, d'une Internet City, troisième zone franche, qui s'ajoute à celles de l'aéroport et de Jebel Ali, vise à faire de Doubaï un centre majeur du commerce électronique mondial. Elle illustre la capacité d'adaptation, voire d'anticipation, de Doubaï .

\section{CONCLUSION}

L'un des aspects les plus frappants de la croissance des villes de la Péninsule Arabique est son caractère planifié. A Doubaï, cette caractéristique est poussée à l'extrême, puisque, non content d'accompagner, voire d'anticiper l'expansion urbaine, l'État, en l'occurrence l'autorité émirale, intervient dans les choix stratégiques qui conditionnent le destin économique de la ville Loin d'être un phénomène purement endogène à relier au génie commerçant de ses habitants, la fortune de Doubaï est due, à la fin du XIXe siècle, à l'implantation par les autorités persanes d'une douane à Lingeh. Cette irruption de l'État dans une région jusque-là ouverte aux échanges conduit de nombreux entrepreneurs iraniens à déplacer leur siège à Doubaï, bourgade connue jusque-là uniquement pour son activité perlière. La présence de cette dynamique communauté immigrée jouera désormais un grand rôle dans le destin économique de la ville. Le pouvoir émiral est détenu par la famille Al-Maktoum, "branche cadette » de la famille régnant sur Abou Dhabi, étroitement soumise à la tutelle britannique qui lui a assuré son autonomie, et alliée à quelques grandes familles négociantes représentant les intérêts commerciaux étrangers. C'est dans ce contexte que le génie de Cheikh Rachid a donné toute sa mesure. Dès avant l'indépendance, celui-ci lutte pour imposer ses idées futuristes, considérées alors comme déraisonnables, qui doivent lui assurer une autonomie stratégique et la possibilité de liaisons directes et modernes avec le monde extérieur. À l'opposé, par exemple, du sultan voisin de Mascate, qui enferme son pays et ses habitants, il obtient les fonds qui lui manquent pour équiper sa modeste capitale d'écoles et d'hôpitaux.

Le pétrole découvert en 1968, puis le quadruplement des cours vont lui apporter les moyens de son ambition, tandis que l'intégration

15. Voir Arabies : "Double casse-tête pour Doubaï ", avril 1999, p. 36 à $39 ; T M E$ : «Salalah Port : a Vision Realised ", janvier 1999, p. 37 à 40. 
obligée dans la Fédération, sous la férule d'Abou Dhabi, va renforcer son sentiment de l'impérative nécessité de préserver la différence de Doubaï. La dynastie régnante a su appréhender les échéances de l'après-pétrole et les possibilités de positionnement de Doubaï de manière à pérenniser la prospérité acquise et de valoriser ses atouts. Elle s'est appuyée sur deux piliers pour mettre en œuvre ses projets : d'une part l'expertise de conseillers occidentaux, surtout anglophones ${ }^{16}$, de l'autre le savoir-faire des grandes familles commerçantes installées sur place.

Un assortiment de moyens, d'objectifs et de cultures différents a permis de faire de Doubaï une ville où l'urbanisme s'ajuste à la gestion de la société et de l'économie. Le succès de Doubaï dépend largement de sa fonctionnalité économique, de la qualité de ses infrastructures et de l'efficacité de leur fonctionnement, du climat de libéralisme et de la flexibilité d'une main-d'œuvre bon marché. Mais elle tient aussi à son paysage urbain attractif pour les élites transnationales, adeptes de la modernité la plus aboutie, comme pour les touristes à haut pouvoir d'achat désireux de conjuguer dépaysement exotique et hébergement luxueux. Cette liberté d'édifier une ville selon des normes de rentabilité et d'efficacité centralisées n'a été possible que parce que la population n'est, pour l'essentiel, que de passage et uniquement implantée pour des raisons professionnelles. La population immigrée n'a ni le droit de posséder du terrain, ni celui de construire et de s'enraciner ; elle doit donc se mouler dans un cadre préparé pour elle, mais établi sans la consulter. C'est en cela, plus que dans son urbanisme qui résulte de ce cadre social et statutaire imposé, que Doubaï n'est au fond pas une ville comme les autres.

\section{BIBLIOGRAPHIE}

E. Ghareeb \& I. Al Abed (eds.), Perspectives on the United Arab Emirates, Trident Press, London, 1997

F.Heard Bey, Les Emirats Arabes Unis, Paris, Karthala, 1999

M. Lavergne, B. Dumortier, «Dubai - von der Wüstenstadt zur Stadt in der Wüste", in: Geographische Rundschau, n9 ${ }^{\circ}$, sept.2000,

16.Pas toujours britanniques (20 000 sujets de la Couronne résident pourtant dans l'émirat) : de l'avis général, le succès de la stratégie de conquête de marchés et de promotion de Doubaï par le Département de l'Aviation Civile, a été le fait de son directeur irlandais et de ses deux assistants connus sous le nom de "Irish Trinity » ((TME, mai 98, p. 37) 
Brunswick, pp. 46-51

R. Marchal, "Doubaï : le développement d'une cité-entrepôt dans le Golfe ", Etudes du CERI, Paris, n²8, 1997

C. Sournia, Femmes des Emirats. Portraits d'hier et de demain, Paris, Albin Michel, 1992

E. Wirth, «Dubai. Ein modernes Städtisches Handels- und Dienstleistungszentrum am Arabischen-Persischen Golf ", in : Mitteilungen der Fränkischen Geographischen Gesellschaft, $\mathrm{n}^{\circ} 48$, Erlangen, 1988 